\title{
Estimación de Edad Mediante la Relación Área Pulpa/Diente en Caninos Mandibulares: Estudio en una Muestra Chilena Utilizando el Método de Cameriere
}

\author{
Dental age Estimation by Pulp/Tooth Ratio in Mandibular Canines: \\ Study in a Chilean Sample by Camerierer's Method
}

Felipe Aguilera-Muñoz'; Agustin Garay -Barrientos ${ }^{2}$; Ignacio Moreno-Lazcano ${ }^{2}$; Pablo Navarro-Cáceres ${ }^{4} \&$ Gabriel M. Fonseca ${ }^{4}$

AGUILERA-MUÑOZ, F.; GARAY-BARRIENTOS, A.; MORENO-LAZCANO, I.; NAVARRO-CÁCERES, P. \& FONSECA, G. M. Estimación de edad mediante la relación área pulpa/diente en caninos mandibulares: Estudio en una muestra Chilena utilizando el método de Cameriere. Int. J. Morphol., 38(2):322-327, 2020.

RESUMEN: La estimación de edad compone un aspecto importante en investigaciones forenses. Diferentes métodos se han descrito en odontología forense basadas en la correlación entre la edad y estructuras dentales. Cameriere et al. proponen un método cuantitativo para estimación de edad en adultos, a partir de la evaluación de la relación del área pulpa/diente, en base a la aposición de dentina secundaria. El objetivo del estudio fue desarrollar modelos de regresión para la estimación de edad dental mediante la relación área pulpa/diente en caninos inferiores en una muestra Chilena. Se analizaron 212 radiografías periapicales digitales (RPD) (86 hombres y 126 mujeres) de caninos mandibulares mediante el programa Image J para establecer el área de la pulpa y el diente. Se registraron los datos de sexo y edad de las RPD seleccionadas en forma ciega. Fueron desarrollados modelos de regresión lineal simples para la estimación de edad. El coeficiente de determinación para R33 fue 27,8 \% y de 29,6 \% para R44, con un error absoluto medio de 11,02 años y 10,37 años respectivamente. El análisis de ANOVA no mostró diferencias estadísticamente significativas para las relaciones área pulpa/diente de caninos según sexo ( $>$ > 0,05). Según los resultados obtenidos, la metodología propuesta por Cameriere et al. es fiable para estimar la edad dental mediante la relación área pulpa/diente en adultos. Sin embargo, en los modelos de regresión desarrollados para la población Chilena, se puede afirmar que el ajuste indicado por los coeficientes de determinación muestran incerteza entre las variables área pulpa/diente y edad cronológica en caninos inferiores, por lo tanto se sugiere considerar otros métodos adicionales para estimar edad en esta población.

PALABRAS CLAVES: Estimación edad; método Cameriere; Odontología forense; Ciencia forense; Relación pulpa/diente.

\section{INTRODUCCIÓN}

La estimación de edad es una tarea dificultosa en contextos de investigación forense (Jeevan et al., 2011) y ha sido utilizada para resolver casos civiles y penales, problemas migratorios, asilo a países extranjeros, entre otros (Schmeling et al., 2008; Azevedo et al., 2015). Para tales fines, se han desarrollado métodos basados en el análisis de distintas porciones anatómicas tales como la carilla articular iliaca (Osborne et al., 2004), la sínfisis púbica (Suchey et al., 1988), las suturas craneales (Garvin \& Passalacqua, 2012), los huesos del carpo (Cameriere et al., 2012a,b) y los dientes (Demirjian et al., 1973). Con respecto a los métodos de corte odontológico que consideran morfología y radiolo- gía dental, en niños han demostrado ser relativamente simples y precisos, basándose principalmente en las etapas del desarrollo y erupción de estos (Pérez et al., 2016). Sin embargo, en adultos esta estimación ha sido un desafío mayor (Jain \& Rai, 2009), ya que los métodos utilizados se basan en procesos degenerativos y/o regresivos con una fuerte influencia ambiental los que han mostrado una menor precisión comparada con los antes mencionados (Solheim \& Vonen, 2006; Limdiwala \& Shah, 2013). En 2004, Cameriere et al. (2004) proponen un método cuantitativo basado la aposición de dentina secundaria, mediante el análisis de la relación área pulpa/diente en radiografías dentales. Dicho mé-

\footnotetext{
${ }^{1}$ Instituto de Odontoestomatología, Universidad Austral de Chile, Valdivia, Chile.

${ }^{2}$ Escuela de Odontología, Facultad de Medicina, Universidad Austral de Chile, Valdivia, Chile.

${ }^{3}$ Centro de investigación en Ciencias Odontológicas (CICO), Facultad de Odontología, Universidad de la Frontera, Temuco, Chile.

${ }^{4}$ Centro de Investigación en Odontología Legal y Forense (CIO), Facultad de Odontología, Universidad de La Frontera, Temuco, Chile.
} 
todo ha sido aplicado utilizando tanto radiografías panorámicas (Jeevan et al. ; Cameriere et al., 2012a,b; Juneja et al., 2014; Apaydin \& Yasar, 2018), como periapicales (Cameriere et al., 2007, 2009; Azevedo et al.; Fabbri et al., 2015) y ha sido estudiado en muestras de varios países como Portugal (Cameriere et al., 2009), Italia (Fabbri et al. ), España (De Luca et al., 2010), India (Jeevan et al.), México (De Luca et al., 2011), Brasil (Azevedo et al.) y Chile (Aguilera-Muñoz et al., 2019), existiendo interés en la validación de este método para distintas poblaciones (Ubelaker \& Parra, 2008; Cunha et al., 2009). Dichos estudios, han considerado frecuentemente el análisis de caninos inferiores, ya que estos dientes son menos afectados por enfermedad periodontal, poseen una menor tasa de extracción y son más resistentes a traumas severos (Padmavati et al., 2011; Kaushal et al., 2003), además de presentar en la mayoría de los casos una sola raíz, lo que favorece realizar mediciones de área para fines de estimación de edad (Cameriere et al., 2007).

Por lo mencionado anteriormente, el objetivo de este estudio es desarrollar modelos de regresión para estimación de edad dental mediante el análisis de la relación de área pulpa/diente en caninos inferiores en una muestra Chilena, utilizando radiografías periapicales digitales (RPD) aplicando el método de Cameriere.

\section{MATERIAL Y MÉTODO}

Se analizaron RPD de caninos mandibulares pertenecientes a 124 individuos residentes en la ciudad de Valdivia (Chile), registradas en el Área de Imagenología de la Clínica Odontológica de la Universidad Austral de Chile, entre los meses de Marzo y Mayo del 2019. Todas las RPD fueron tomadas según las normas de la Técnica del Paralelismo (Goaz \& White, 1995), utilizando posicionador Rinn XCP (FPS 3000 Film Positioning System XCP), con el fin de reducir la distorsión de imagen y estandarizar los registros.
Junto a las RPD, se consignaron los datos de sexo, fecha de nacimiento y de la toma radiográfica, con el fin de determinar la edad cronológica de los participantes. Estos datos fueron anonimizados y cada individuo fue designado con un código a los fines de protección de identidad. El protocolo de investigación fue aprobado por el Comité de Ética Científico del Servicio de Salud de Valdivia.

Para este estudio, fueron incluidos dientes sanos e imágenes radiográficas que contasen con los criterios mínimos de calidad propuestos por Villanueva \& Fonseca (2011). Se excluyeron radiografías presentando dientes con malformaciones coronarias o radiculares, procesos patológicos con pérdidas de sustancia (caries o fracturas), deformaciones camerales, restauraciones, aparatología de ortodoncia u otra condición que afectase potencialmente el análisis métrico del área pulpa u área diente, siguiendo los estándares desarrollados por Aguilera-Muñoz et al.

Fue utilizada la nomenclatura propuesta por la Fédération Dentaire Internationale (FDI) y la relación área pulpa/diente fue denominada como " $R$ ". Así, la relación área pulpa/diente correspondiente al canino inferior izquierdo fue denominada "R33" y para el canino inferior derecho "R43".

Todas las radiografías utilizadas fueron importadas en formato JPEG al programa ImageJ (National Institutes of Health, Bethesda, MD, USA) y el procesamiento de imágenes para establecer la relación área pulpa/diente, fue realizado según lo establecido por Cameriere et al. (2007), el cual considera mediciones del área en pixeles tanto de la superficie total del diente como también de la cámara pulpar. Las regiones mencionadas fueron seleccionadas mediante la opción "Polygon Selection Tool" (Fig. 1), y las áreas correspondientes fueron obtenidas mediante la función "Set Measurements" en la sección "Results". Cada radiografía periapical fue numerada en forma correlativa sin que los observadores conocieran la edad cronológica de los individuos.

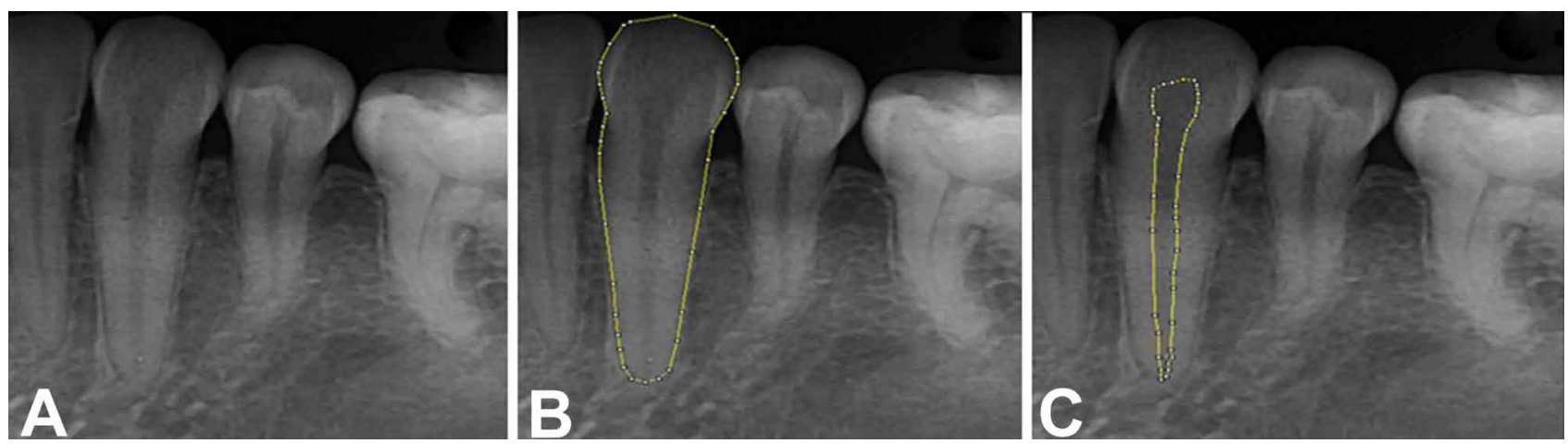

Fig. 1. Imagen radiográfica del canino inferior izquierdo (33) (a), medición del área del diente (b), medición del área de la pulpa (c), utilizando ImageJ. 
Para evaluar la concordancia de las observaciones se analizó una muestra aleatoria de 20 radiografías en un intervalo de 2 semanas. Todas las mediciones fueron realizadas por dos observadores (A.G.B., I.M.L.). Para la valoración de la reproducibilidad de las observaciones, tres observadores repitieron el mismo proceso (F.A.M., A.G.B., I.M.L.). Se calculó el Coeficiente de Correlación Intraclase (ICC) considerando: $<0$ sin acuerdo, $<0,4$ pobre, $0,4-0,59$ moderado, 0,6-0,74 bueno, >0,74 excelente, según lo propuesto por Fleiss (1981).

Fueron incluidas 212 radiografías periapicales que cumplieron con los criterios de inclusión antes señalados. Del total de radiografías, 86 correspondieron a hombres $(40,6$ $\%)$ y 126 a mujeres $(59,4 \%)$.

Los datos fueron tabulados en una plantilla de Google Sheets, para luego ser importados al programa IBM SPSS (v.23.0), estableciéndose un un nivel de significancia estadística de $\mathrm{p}<0,05$.
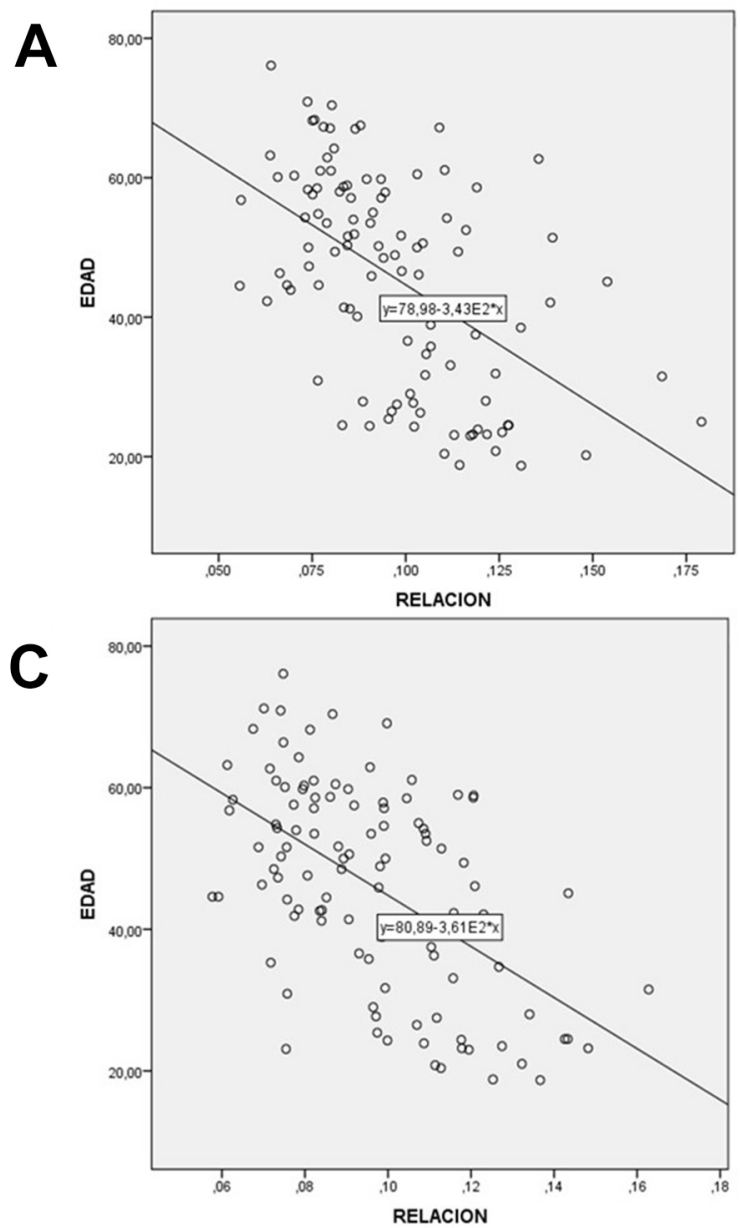

Se desarrollaron modelos de regresión lineal simples para estimación de edad dental, los que fueron comparados según sexo y relación de caninos mediante el análisis de ANOVA, calculando además los parámetros de Coeficiente de determinación (R2), error estándar (SE) y error absoluto medio (EM).

\section{RESULTADOS}

No existieron diferencias estadísticamente significativas al realizar las mediciones intra e interobservador con un ICC >0,81, lo cual reveló un grado de confiabilidad excelente, según lo establecido por Fleiss.

El rango de edad varió entre los 18 y 76 años, con una edad media de 45,99 ( $\pm 18,88)$ años. En la mayoría de los grupos de edad, la distribución entre hombres y mujeres fue homogénea, a excepción del grupo entre 49 y 58 años.
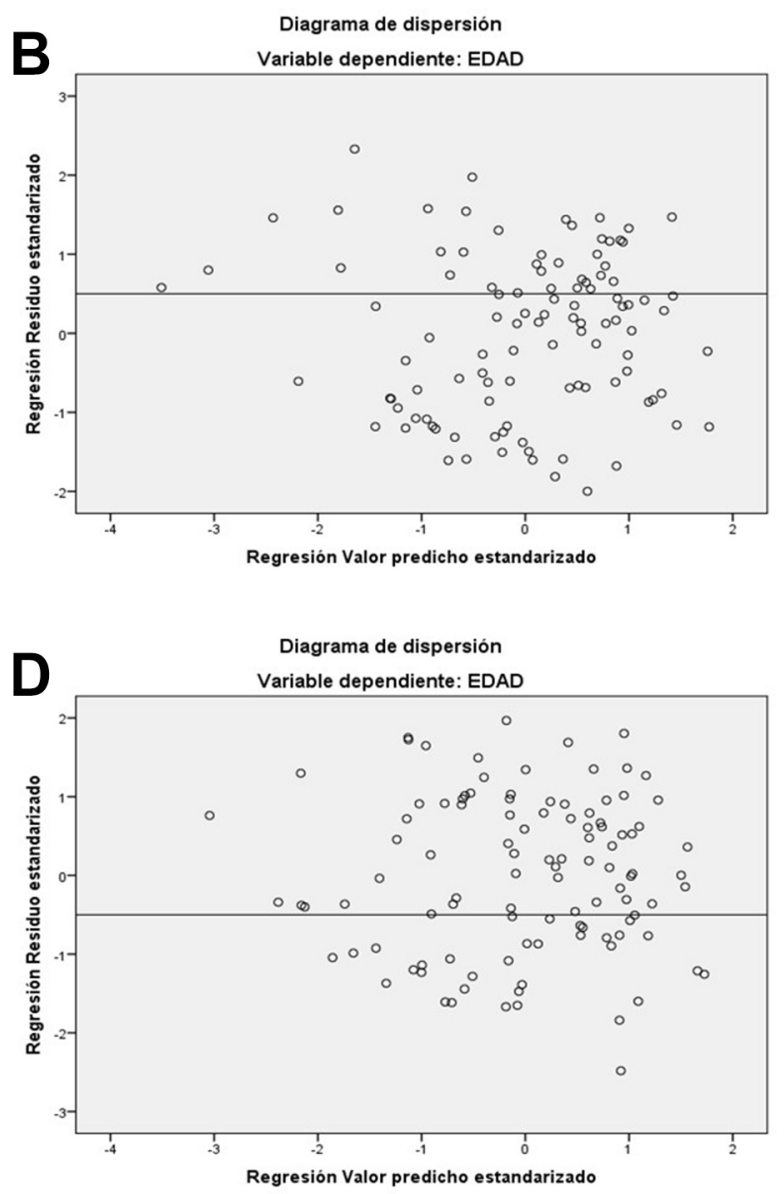

Fig. 2. Gráfica de dispersión (panel izquierdo) y residuos contra valores ajustados (panel derecho) usando un modelo de regresión lineal simple. Para relaciones R33 (a,b) y R43(c,d). 
Los detalles de la distribución según sexo y edad se observan en la Tabla I.

La relación de área pulpa/diente para los caninos disminuyó con la edad, y se obtuvieron valores para R33 entre 0,05 a 0,17 , y para $\mathrm{R} 43$ estos valores variaron de 0,05 a 0,16. La Figura 2, muestra las relaciones en los diagramas de regresión y residuos para R33 y R43.

En los análisis de regresión desarrollados, se obtuvieron valores de R2 de 27,8 \%, para R33 y de 29,6\% para $\mathrm{R} 43$, con valores error estandar de 12,9 años para R33 y de
12,3 años para R43. Los valores de error absoluto medio fueron de 11,02 para R33 y de 10,37 para R43. Cuando se tuvo en cuenta la relación área pulpa / diente en R33, se obtuvo la siguiente ecuación de regresión lineal: Edad= 78,98-343,399*R33. Por otra parte, para R43 la ecuación fue: $\mathrm{Edad}=80,886-360,910$ R43. Los detalles de muestran en la Tabla II.

No existieron diferencias significativas al realizar el análisis de ANOVA para las relaciones área pulpa/diente de caninos según sexo ( $p>0,05)$.

Tabla I. Distribución de edad y sexo para las muestras de radiografías periapicales.

\begin{tabular}{lccccccc}
\hline Sexo & \multicolumn{7}{c}{ Edad de corte } \\
\hline & $18-28$ & $29-38$ & $39-48$ & $49-58$ & $59-68$ & $>69$ & Total \\
\hline Hombre & 19 & 11 & 19 & 19 & 14 & 4 & 86 \\
Mujer & 25 & 13 & 21 & 42 & 21 & 4 & 126 \\
Total & 44 & 24 & 40 & 61 & 35 & 8 & 212 \\
\hline
\end{tabular}

Tabla II. Ecuación de regresión utilizando premolares y caninos inferiores.

\begin{tabular}{llcccc}
\hline Relación & Ecuación & $\mathrm{R}^{2}$ & $\mathrm{SE}$ & $\mathrm{EM}$ & $\mathrm{p}$ \\
\hline R33 & edad=78,98-343,399x & 0,27 & 12,98 & 11,02 & 0.01 \\
R43 & edad=80,886-360,910x & 0,29 & 12,30 & 10,37 & 0.01 \\
\hline
\end{tabular}

R2, coeficiente de determinación; SE, error estándar; EM, error absoluto medio; $p$, significancia estadística.

\section{DISCUSIÓN}

La estimación de edad ha sido considerada un aspecto importante al establecer un perfil en investigaciones forenses (Cameriere et al., 2009; Jeevan et al.). Para tales fines, han sido estudiadas diferentes porciones anatómicas entre las que la estructura dental ha ofrecido una buena confiabilidad. En este estudio se incluyeron caninos inferiores, por ser los dientes menos afectados por injurias a través del tiempo (Kaushal et al.; Padmavati et al. ) facilitando con ello la medición de las áreas correspondientes.

En este estudio, al igual que en el estudio de Cameriere et al. (2007) se utilizaron radiografías periapicales debido a la menor superposición de estructuras y distorsión de la imágen en comparación con las radiografías panorámicas (Cameriere et al., 2007, 2009). Por otra parte, la radiografía digital ayudaría a realizar los análisis de estas relaciones de mejor forma, además de generar menor exposición a radiación por parte del paciente (Jeevan et al.; Meinl et al., 2008).
Cabe señalar que el método utilizado para realizar mediciones en las RPD, obtuvo un alto grado de acuerdo intra e interobservador, al igual que lo reportado por Aguilera-Muñoz et al., Juneja et al., Cameriere et al. (2013), De Luca et al. (2011), lo que señala una alta reproducibilidad del método y, por ende, una buena replicabilidad de las mediciones por parte de otros observadores.

Con respecto al coeficiente de determinación, en este estudio se obtuvieron valores bajos, con cifras de 27,8\% para R33 y 29,6 \% para R43, lo que indicó una baja correlación entre los modelos obtenidos a diferencia de lo reportado por Cameriere et al. $(2007,2009)$ y Fabbri et al., quienes obtienen un coeficiente de determinación de $86 \%, 93 \%$ y $93 \%$ respectivamente. Al igual que en nuestro estudio, estos reportes utilizaron RPD de caninos inferiores señalando que existe una relación inversa entre la edad y la relación pulpa/diente, lo que también fue identificado en los resultados obtenidos. 
El estudio original de Cameriere et al. (2004) mostró altos niveles de predicción de la edad, revelando que existe una relación lineal entre la edad cronológica y la relación área pulpa/diente en caninos superiores. Si bien los resultados de nuestro estudio siguen un patrón de tendencia lineal, el modelo estudiado no se ajusta de buena forma a la tendencia de los datos, logrando niveles de predicción por bajo de lo esperado.

Dado que algunos estudios han recomendado ajustes y formulas especificas para cada población debido a amplias variaciones (Ubelaker \& Parra; Cunha et al.), se desarrollaron fórmulas específicas para esta población, obteniendo ecuaciones de regresión lineal para ambos caninos mandibulares, sin efecto significativo del sexo en los modelos, al igual que en reportes previos (Cameriere et al., 2007, 2009; De Luca et al., 2010; Jeevan et al.). Sin embargo, los modelos de regresión obtenidos no se ajustaron bien a la tendencia de los datos, al igual que reportes como el de Babshet et al. (2010) en una población India. Esto podría ser atribuido a una serie de factores, como son la diferencia étnica y la variación en el patrón de depósito de dentina secundaria en la población Chilena, en comparación con la muestra Italiana original (Cameriere et al., 2007). Otra razón, podría atribuirse a que la mayoría de los estudios utilizaron colecciones osteológicas como muestras (Cameriere et al., 2004, 2007, 2009), sin la dificultad de la toma de radiografías de caninos mandibulares en boca.

Dentro de las limitantes del estudio, puede mencionarse la selección de la muestra por conveniencia, al igual que en estudios previos (Cameriere et al., 2009; Babshet et al.) donde se utilizaron tamaños muestrales similares. Por otra parte, la heterogeneidad de la muestra podría afectar los resultados obtenidos al igual que la dificultad de realizar ciertas mediciones con exactitud debido a la calidad de las imágenes proporcionadas. Futuras investigaciones deberían enfocarse en establecer un buen cálculo muestral según la población en estudio y validar las ecuaciones obtenidas. Además, se sugiere añadir más dientes a la medición y realizar regresiones múltiples, con el fin de evaluar si la la estimación de edad es más precisa.

\section{CONCLUSIONES}

La metodología propuesta por Cameriere et al. (2004) es fiable para establecer relaciones entre el área pulpa/diente en caninos inferiores. Sin embargo, según los resultados obtenidos en los modelos de regresión desarrollados para la población Chilena, se puede afirmar que el ajuste indicado por los coeficientes de determinación muestran incerteza entre las variables área pulpa/diente y edad cronológica en caninos inferiores, por lo tanto se sugiere considerar otros métodos adicionales para estimar edad en esta población.

\section{AGRADECIMIENTOS}

Esta investigación está basada en una tesis realizada por Agustin Garay Barrientos e Ignacio Moreno Lazcano como requerimiento para la obtención del grado académico de Licenciado en Odontología de la Universidad Austral de Chile, Julio, 2019.

AGUILERA-MUÑOZ, F.; GARAY-BARRIENTOS, A.; MORENO-LAZCANO, I.; NAVARRO-CÁCERES, P. \& FONSECA, G. M. Dental age Estimation by pulp/tooth ratio in mandibular canines: Study in a Chilean sample by Cameriere's method. Int. J. Morphol., 38(2):322-327, 2020.

SUMMARY: Age estimation is an important aspect In forensic investigations. Different methods in forensic odontology based on the correlation between age estimation in adults, from the analysis of the pulp/tooth area, based on the apposition of secondary dentine. The aim of the study was to develop regression models for the dental age estimation by the relation pulp/tooth area, in lower canines in a Chilean sample, using digital peri-apical radiographs (DPR) applying Cameriere's method. We analyzed 212 DPR (86 males and 126 females) mandibular canines through Image J program to measure the pulp/tooth area. Age and sex information was obtained of the DPR's blindly selected. We developed simple linear regression models for age estimation. The coefficient of determination to R33 was R2 age and dental structures have been described. Cameriere $e t$ $a l$. proposed a quantitative method for $27.8 \%$ and R2 $29.6 \%$ to R44, with a mean absolute error of 11.02 years, to R33 and 10.37 years to R44. ANOVA analysis showed no statistically significant differences for the pulp/tooth relation area of canines according to sex ( $p>0.05)$. According to the results, the Cameriere's et al., method is reliable for dental age estimation according to pulp/tooth ratio in adults. However, in the regression models developed for Chilean population, it can be stated that the adjustment indicated by the coefficients of determination, show uncertainty between the pulp / tooth area and chronological age in lower canines, therefore it is suggested to use additional estimation methods for age in this population.

KEY WORDS: Age determination; Pulp/tooth area; Forensic science; Forensic odontology; Cameriere's method.

\section{REFERENCIAS BIBLIOGRAFICAS}

Aguilera-Muñoz, F.; López-Lázaro, S.; De-Luca, S.; Navarro-Cáceres, P. \& Fonseca, G. M. Dental age estimation in adults by pulp/tooth ratio in lower premolars and canines by orthopantomographs, in a chilean sample. Int. J. Morphol., 37(3):959-64, 2019. 
Apaydin, B. K. \& Yasar, F. Accuracy of the demirjian, willems and cameriere methods of estimating dental age on turkish children. Niger. J. Clin. Pract., 21(3):257-63, 2018

Azevedo, A. C. S.; Alves, N. Z.; Michel-Crosato, E.; Rocha, M.; Cameriere, R. \& Biazevic, M. G. H. Dental age estimation in a Brazilian adult population using Cameriere's method. Braz. Oral Res., 29(1):1-9, 2015.

Babshet, M.; Acharya, A. B. \& Naikmasur, V. G. Age estimation in Indians from pulp/tooth area ratio of mandibular canines. Forensic Sci. Int., 197(1-3):125.e1-4, 2010.

Cameriere, R.; Cunha, E.; Sassaroli, E.; Nuzzolese, E \& Ferrante, L. Age estimation by pulp/tooth area ratio in canines: study of a Portuguese sample to test Cameriere's method. Forensic Sci. Int., 193(1-3):128.e16, 2009.

Cameriere, R.; De Luca, S.; Alemán, I.; Ferrante, L. \& Cingolani, M. Age estimation by pulp/tooth ratio in lower premolars by orthopantomography. Forensic Sci. Int., 214(1-3):105-12, 2012.

Cameriere, R.; De Luca, S.; Biagi, R.; Cingolani, M.; Farrorato, G. \& Ferrante, L. Accuracy of three age estimation methods in children by measurements of developing teeth and carpals and epiphyses of the ulna and radius. J. Forensic Sci., 57(5):1263-70, 2012.

Cameriere, R.; Ferrante, L. \& Cingolani, M. Variations in pulp/tooth area ratio as an indicator of age: a preliminary study. J. Forensic Sci., 49(2):317-9, 2004.

Cameriere, R.; Ferrante, L.; Belcastro, M. G.; Bonfiglioli, B.; Rastelli, E. \& Cingolani, M. Age estimation by pulp/tooth ratio in canines by periapical x-rays. J. Forensic Sci., 52(1):166-70, 2007.

Cunha, E.; Baccino, E.; Martrille, L.; Ramsthaler, F.; Prieto, J.; Schuliar, Y.; Lynnerup, N. \& Cattaneo, C. The problem of aging human remains and living individuals: a review. Forensic Sci. Int., 193(1-3):1-13, 2009.

De Luca, S.; Alemán, I.; Bertoldi, F.; Ferrante, L.; Mastrangelo, P.; Cingolani, M. \& Cameriere, R. Age estimation by tooth/pulp ratio in canines by peri-apical X-rays: reliability in age determination of Spanish and Italian medieval skeletal remains. J. Archaeol. Sci., 37(12):3048$58,2010$.

De Luca, S.; Bautista, J.; Alemán, I. \& Cameriere, R. Age-at-death estimation by pulp/tooth area ratio in canines: study of a 20th-century Mexican sample of prisoners to test Cameriere's method. J. Forensic Sci., 56(5):1302-9, 2011.

Demirjian, A.; Goldstein, H. \& Tanner, J. M. A new system for dental age assessment. Hum. Biol., 45(2):211-27, 1973.

Fabbri, P. F.; Viva, S.; Ferrante, L.; Lonoce, N.; Tiberi, I. \& Cameriere, R. Radiological tooth/pulp ratio in canines and individual age estimation in a sample of adult neolithic skeletons from Italy. Am. J. Phys. Anthropol., 158(3):423-30, 2015.

Fleiss, J. L. Statistical Methods for Rates and Proportions. New York, John Wiley, 1981.

Garvin, H. M. \& Passalacqua, N. V. Current practices by forensic anthropologists in adult skeletal age estimation. J. Forensic Sci., 57(2):427-33, 2012.

Goaz, P. W. \& White, S. C. Radiología Oral. 3ª ed. Madrid, Mosby, 1995. pp. $153-220$

Jain, R. K. \& Rai, B. Age estimation from permanent molar's attrition, of Haryana population. Indian J. Forensic Odontol., 2(2):59-61, 2009.

Jeevan, M. B.; Kale, A. D.; Angadi, P. V. \& Hallikerimath, S. Age estimation by pulp/tooth area ratio in canines: Cameriere's method assessed in an Indian sample using radiovisiography. Forensic Sci. Int., 204(13):209.e1-5, 2011.

Juneja, M.; Devi, Y. B. K.; Rakesh, N. \& Juneja, S. Age estimation using pulp/tooth area ratio in maxillary canines-A digital image analysis. $J$. Forensic Dent. Sci., 6(3):160-5, 2014.

Kaushal, S.; Patnaik, V. V. G. \& Agnihotri, G. Mandibular canines in sex determination. J. Anat. Soc. India, 52(2):119-24, 2003.

Limdiwala, P. G. \& Shah, J. S. Age estimation by using dental radiographs. J. Forensic Dent. Sci., 5(2):118-22, 2013.

Meinl, A.; Huber, C. D.; Tangl, S.; Gruber, G. M.; Teschler-Nicola, M. \& Watzek, G. Comparison of the validity of three dental methods for the estimation of age at death. Forensic Sci. Int., 178(2-3):96-105, 2008.

Osborne, D. L.; Simmons, T. L. \& Nawrocki, S. P. Reconsidering the auricular surface as an indicator of age at death. J. Forensic Sci., 49(5):905$11,2004$.

Padmavati, K.; Farah, V. M.; Syed, A. A. \& Ather, S. A. Mandibular canine index: A tool for sex determination. J. Indian Dent. Assoc., 5:18, 2011.

Pérez, M.; Herrera, A.; Moreno, S. \& Moreno, F. Estimación de la edad dental a través de seis métodos radiográficos en un grupo de afrodescendientes y mestizos caucasoides. Cuad. Med. Forense, 22(34):81-92, 2016.

Schmeling, A.; Grundmann, C.; Fuhrmann, A.; Kaatsch, H. J.; Knell, B.; Ramsthaler, F.; Reisinger, W.; Riepert, T.; Ritz-Timme, S.; Rösing, F. $\mathrm{W}$; et al. Criteria for age estimation in living individuals. Int. J. Legal Med., 122(6):457-60, 2008.

Solheim, T. \& Vonen, A. Dental age estimation, quality assurance and age estimation of asylum seekers in Norway. Forensic Sci. Int., 159 Suppl. 1:S56-60, 2006.

Suchey, J. M.; Brooks, S. T. \& Katz, D. Instructions for Use Of The Suchey Brooks System for Age Determination of the Female Os Pubis. Instructional Materials Accompanying Female Pubic Symphyseal Models of the Suchey-Brooks System. Colorado, France Casting, 1988.

Ubelaker, D. H. \& Parra, R. C. Application of three dental methods of adult age estimation from intact single rooted teeth to a Peruvian sample. J. Forensic Sci., 53(3):608-11, 2008.

Villanueva, J. C. \& Fonseca, G. M. Digitalización de radiografías panorámicas en la rutina de odontólogos de la Provincia de Córdoba, Argentina. Aseguramiento de calidad para procedimientos de identificación forense. F. O. P. J., 2(5):10-4, 2011.

Dirección para correspondencia:

Felipe Aguilera Muñoz

Instituto de Odontoestomatología

Facultad de Medicina

Universidad Austral de Chile

Valdivia

CHILE

ORCID: 0000-0002-0390-0018

E-mail: felipe.aguilera@uach.cl

Recibido : 17-07-2019

Aceptado: 08-10-2019 